\title{
Syring, Marcus
}

\section{Videobasierte Kasuistik in der Lehre}

Wittek, Doris [Hrsg.]; Rabe, Thorid [Hrsg.]; Ritter, Michael [Hrsg.]: Kasuistik in Forschung und Lehre. Erziehungswissenschaftliche und fachdidaktische Ordnungsversuche. Bad Heilbrunn : Verlag Julius Klinkhardt 2021, S. 230-244

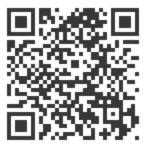

Quellenangabe/ Reference:

Syring, Marcus: Videobasierte Kasuistik in der Lehre - In: Wittek, Doris [Hrsg.]; Rabe, Thorid [Hrsg.]; Ritter, Michael [Hrsg.]: Kasuistik in Forschung und Lehre. Erziehungswissenschaftliche und fachdidaktische Ordnungsversuche. Bad Heilbrunn : Verlag Julius Klinkhardt 2021, S. 230-244 - URN: urn:nbn:de:0111-pedocs-215708 - DOI: 10.25656/01:21570

https://nbn-resolving.org/urn:nbn:de:0111-pedocs-215708

https://doi.org/10.25656/01:21570

in Kooperation mit / in cooperation with:

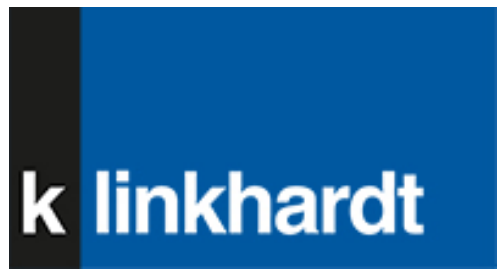

http://www.klinkhardt.de

\section{Nutzungsbedingungen}

Dieses Dokument steht unter folgender Creative Commons-Lizenz: http://creativecommons.org/licenses/by-nc-sa/4.0/deed.de - Sie dürfen das Werk bzw. den Inhalt unter folgenden Bedingungen vervielfältigen, verbreiten und öffentlich zugänglich machen sowie Abwandlungen und Bearbeitungen des Werkes bzw. Inhaltes anfertigen: Sie müssen den Namen des Autors/Rechteinhabers in der von ihm festgelegten Weise nennen. Dieses Werk bzw. der Inhalt darf nicht für kommerzielle Zwecke verwendet werden. Die neu entstandenen Werke bzw. Inhalte dürfen nur unter Verwendung von Die neu entstandenen Werke bzw. Inhalte dürfen nur unter Verwendung von
Lizenzbedingungen weitergegeben werden, die mit denen dieses Lizenzvertrages identisch oder vergleichbar sind.

Mit der Verwendung dieses Dokuments erkennen Sie die Nutzungsbedingungen an.

\section{Terms of use}

This document is published under following Creative Commons-License: http://creativecommons.org/licenses/by-nc-sa/4.0/deed.en - You may copy, distribute and transmit, adapt or exhibit the work in the public and alter, transform or change this work as long as you attribute the work in the manner specified by the author or licensor. You are not allowed to make commercial use of the work. If you alter, transform, or change this work in any way, you may distribute the resulting work only under this or a comparable license.

By using this particular document, you accept the above-stated conditions of use.

\section{Kontakt / Contact:}

\section{peDOCS}

DIPF | Leibniz-Institut für Bildungsforschung und Bildungsinformation Informationszentrum (IZ) Bildung

E-Mail: pedocs@dipf.de

Internet: www.pedocs.de

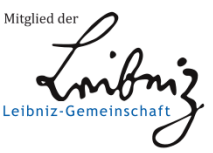




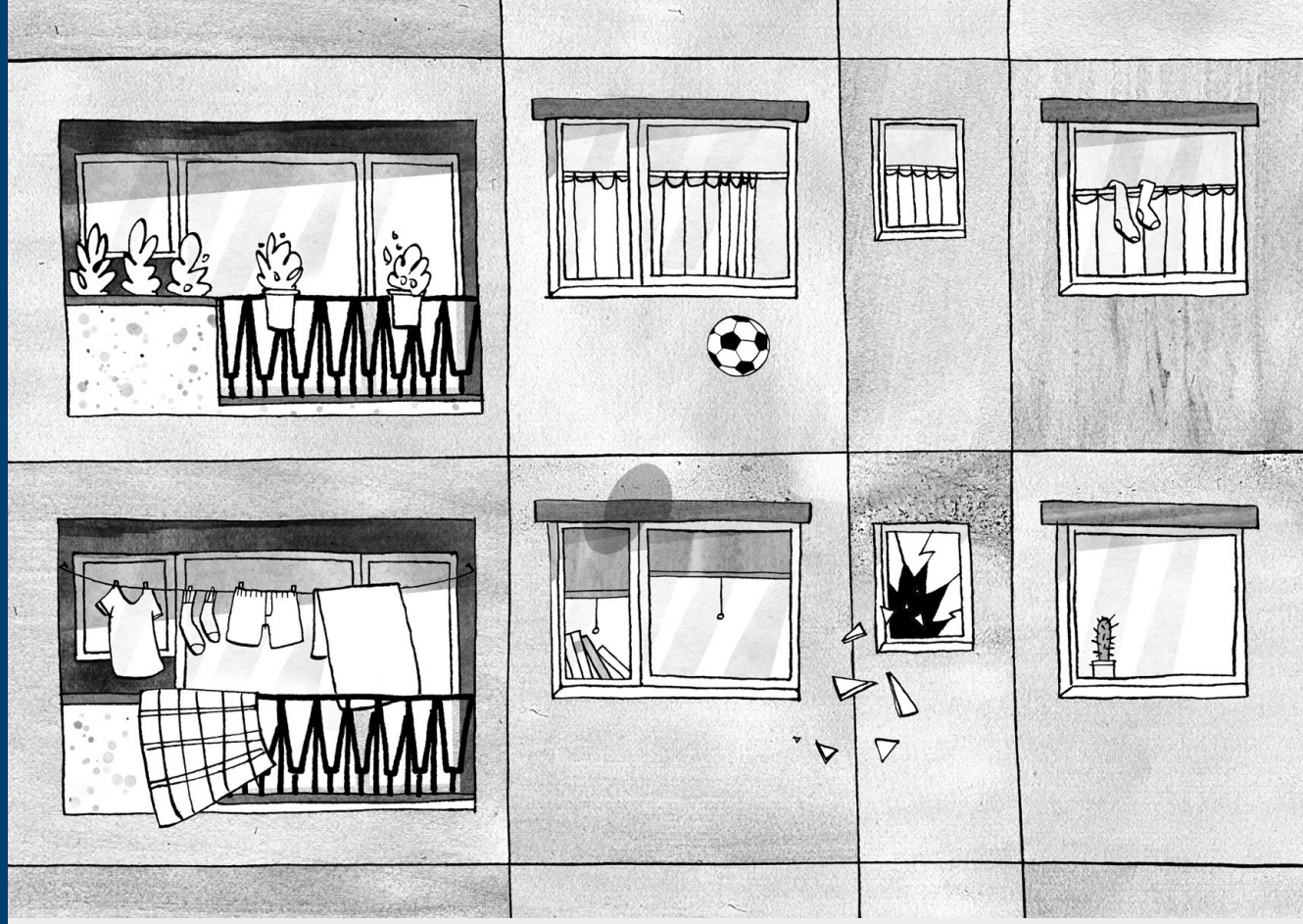

Doris Wittek

Thorid Rabe

Michael Ritter

(Hrsg.)

Kasuistik in Forschung

und Lehre

Erziehungswissenschaftliche und

fachdidaktische Ordnungsversuche 


\section{Doris Wittek Thorid Rabe Michael Ritter (Hrsg.)}

\section{Kasuistik in Forschung und Lehre}

Erziehungswissenschaftliche und fachdidaktische Ordnungsversuche 
Das Projekt KALEI (Kasuistische Lehrerbildung für inklusiven Unterricht), in dessen Rabmen diese Publikation entstanden ist, wird im Rahmen der gemeinsamen „Qualitätsoffensive Lehrerbildung "von Bund und Ländern aus Mitteln des Bundesministeriums für Bildung und Forschung unter dem Förderkennzeichen 01JA1618 gefördert.

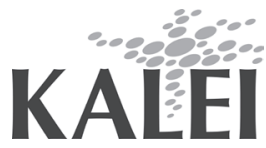

GEFÖRDERT VOM

Bundesministerium

für Bildung

und Forschung

Dieser Titel wurde in das Programm des Verlages mittels eines Peer-Review-Verfahrens aufgenommen. Für weitere Informationen siehe www.klinkhardt.de.

Bibliografische Information der Deutschen Nationalbibliothek Die Deutsche Nationalbibliothek verzeichnet diese Publikation in der Deutschen Nationalbibliografie; detaillierte bibliografische Daten sind im Internet abrufbar über http://dnb.d-nb.de.

2021.ng. () by Julius Klinkhardt.

Zeichnung Umschlagseite 1: () Wolfgang Philippi, Wuppertal (www.wolfgangphilippi.de).

Druck und Bindung: AZ Druck und Datentechnik, Kempten.

Printed in Germany 2021.

Gedruckt auf chlorfrei gebleichtem alterungsbeständigem Papier.

(c) (i)(9) Die Publikation (mit Ausnahme aller Fotos, Grafiken und Abbildungen) ist veröffentlicht unter der Creative Commons-Lizenz: CC BY-NC-SA 4.0 International https://creativecommons.org/licenses/by-nc-sa/4.0/ 


\section{Inhalt}

Doris Wittek, Thorid Rabe, Michael Ritter

Kasuistik in Forschung und Lehre - Ordnungen und Unordnungen

mit Blick auf die Disziplinen

Fälle disziplinär diskutieren - Fälle interdisziplinär verorten

Merle Hummrich

Der Fall aus der Perspektive der Erziehungswissenschaft

Friederike Heinzel

Der Fall aus der Perspektive von Schulpädagogik und

Lehrer*innenbildung: Ein Ordnungsversuch 41

\section{Ursula Bredel, Irene Pieper}

Der Fall aus der Perspektive der Fachdidaktik:

Fachliche Lernprozesse als Ziel und Ausgangspunkt

Jörg Dinkelaker

Der Fall aus der Perspektive der Erwachsenenpädagogik:

Fälle als Grenzobjekte zwischen erziehungswissenschaftlicher und pädagogischer Praxis

Diana Handschke, Bettina Hünersdorf

Der Fall aus der Perspektive der Sozialpädagogik: Fallverstehen als

Kristallisationspunkt zwischen Profession,

Disziplin und dem Politischem 108

\section{Mit Fällen forschen - durch Fälle Wissen schaffen}

\section{Carla Schelle}

Funktionen von Fallarbeit in der Forschung: Fallverständnisse und

Darstellungsweisen aus der Schul- und Unterrichtsforschung

Werner Helsper

Der Fall in der wissenschaftlichen Erkenntnisbildung:

Ein Ordnungsversuch und Konsequenzen für die

Lehrerprofessionalisierung 
6 Inhaltsverzeichnis

Mit Fällen lehren - an Fällen lernen

Richard Schmidt, Doris Wittek

Ziele und Modi von Fallarbeit in der universitären Lehre

Tobias Leonhard

Der Fall in den Schul- bzw. Berufspraktischen Studien

Olaf Krey, Thorid Rabe, Michael Ritter

Fallarbeit in den Fachdidaktiken: Eine analytische Auseinandersetzung

mit Studienelementen der Physik- und Deutschdidaktik

Marcus Syring

Videobasierte Kasuistik in der Lehre

Benjamin Krasemann

Zur Archivierung von Fällen

(Quer-)Perspektiven eröffnen - (Un-)Ordnungen neu denken

Richard Schmidt, Doris Wittek

Rekonstruktive Kasuistik - ein unerreichbares Ideal universitärer Lehre.

Empirische Hinweise zum Widerstreit von Programmatik und Praxis

Thomas Wenzl

Der Fall als Reflexionsübung? Oder: Die erziehungswissenschaftliche

Kasuistik im Lichte der Fallarbeit im Studium der Jurisprudenz und

der Medizin

Andreas Wernet

Fallstricke der Kasuistik

Autor*innenverzeichnis 
Marcus Syring

\section{Videobasierte Kasuistik in der Lehre}

Ein Blick auf die aktuell geförderten Projekte im Rahmen der Qualitätsoffensive Lehrerbildung (vgl. Ramboll 2018) lässt erahnen, welche Bedeutung dem Einsatz von Unterrichtsvideos im Rahmen der universitären Lehre derzeit beigemessen wird. Dies hängt auch damit zusammen, dass es die neuen technischen Möglichkeiten einfacher (und auch günstiger) machen (vgl. Petko \& Reusser 2004), verstärkt Unterrichtsausschnitte in Form von Videos oder Filmen in der Lehrer*innenbildung einzusetzen (vgl. Moreno \& Valdez 2007; Sherin 2007; Krammer 2014). Auch die hierzu gesondert erschienenen Themenschwerpunkte der Beiträge zur Lehrerbildung (Heft 2, 2014) sowie der Unterrichtswissenschaft (Heft 4, 2016) unterstreichen diese Bedeutungszunahme. Diesen großen Hoffnungen steht aber dennoch ein - trotz technischem Fortschritt - nicht geringer Aufwand gegenüber, vor allem bei der Erstellung von Unterrichtsvideos: Kosten und Nutzen von Videoaufzeichnungen im (eigenen) Unterricht gilt es hierbei genau abzuwägen, da technische (Kamera(s), Ton etc.), personelle (Wer filmt?) und organisatorische (bspw. Einverständniserklärungen der Eltern) Aspekte zu berücksichtigen sind. Zudem stellen sich ethische Fragen, z.B. mit Blick auf die Anonymität und die Speicherung der Daten.

Nichtsdestotrotz gelten Videos häufig als ein zentrales „Erschließungsmedium“ (Dorlöchter u.a. 2013, 6), um Unterricht in der Lehrer*innenbildung und -fortbildung exemplarisch zu illustrieren, um als Medium für fallbasiertes Arbeiten im Sinne des Kompetenzerwerbs zu gelten oder um als Mittel zur Ausprägung von Reflexionsfähigkeit zu dienen (vgl. zu unterschiedlichen Zielen des Videoeinsatzes den Review von Gaudin \& Chaliès 2015). In allen drei genannten Funktionen wird deutlich, welches Professions- und Professionalisierungsverständnis vielen Ansätzen der videobasierten Kasuistik in der Lehrer*innenbildung zu Grunde liegt: der kompetenztheoretische Ansatz, wie ihn beispielweise Baumert und Kunter (2006) in Anlehnung an Shulmann (1986) entwickeln. Demnach ist - verkürzt gesagt - der Lehrer*innenberuf durch ein bestimmtes Set an erlernbaren Kompetenzen, Überzeugungen, motivationalen Orientierungen und selbstregulativen Fähigkeiten gekennzeichnet. Diese Kompetenzen gilt es - aus Perspektive dieses Professionalisierungsverständnisses - mithilfe von Unterrichtsvideos bereits in der ersten Phase der Lehrer*innenbildung anzubahnen und später (Referendariat, Berufsleben) immer wieder mithilfe von Unterrichtsvideos zu analysieren, zu reflektieren und ggf. zu verändern. 
Der folgende Beitrag versucht Ordnung in die vielfältigen Einsatzmöglichkeiten, aber auch Forschungsbefunde zur videobasierten Kasuistik in der Lehrer*innenbildung zu bringen. Dabei werden folgende Ordnungsversuche unternommen:

- Erster Ordnungsversuch: Historische Entwicklung videobasierter Kasuistik

- Zweiter Ordnungsversuch: Ziele videobasierter Kasuistik - Was ist der Fall?

- Dritter Ordnungsversuch: Wirkungen videobasierter Kasuistik in der Lehrer*innenbildung

- Vierter Ordnungsversuch: Merkmale von videobasierten Lernumgebungen

Der Beitrag schließt mit didaktischen Empfehlungen zur Gestaltung videobasierter kasuistischer Lehre.

\section{Erster Ordnungsversuch: Historische Entwicklung videobasierter Kasuistik}

Unterrichtsvideos werden aktuell zwar verstärkt in der Lehrer*innenbildung eingesetzt, neu ist das Medium jedoch nicht. Bereits seit den frühen 1960er-Jahren wurden Videos in den USA im Rahmen von Microteaching und Interaction Analysis eingesetzt, später dann in Formaten des Modelling Expert Teaching sowie seit den 1980er-Jahren als sogenannte video-based cases. Parallel dazu entwickelte sich in Deutschland seit den 1960er-Jahren das Konzept der Unterrichtsmitschau. Während in den 1980er- und 1990er-Jahren das fallbasierte Lernen mit Texten (vgl. Shulman 1992) fokussiert wurde, gelangten seit den 2000er-Jahren Videos stärker in den Fokus (vgl. Goldman 2007). Unterrichtsvideos kamen zunächst vor allem im Bereich der Fortbildung von Lehrkräften zum Einsatz (z.B. um eigenen oder fremden Unterricht zu analysieren) und hier wiederum vorrangig im mathematisch-naturwissenschaftlichen Bereich. Nach und nach hielt die videobasierte Kasuistik dann auch in die Ausbildungskontexte von Lehramtsstudierenden Einzug (vgl. Santagata \& Guarino 2011; Baecher u.a. 2018), sowohl in den Bildungswissenschaften als auch in den Fachdidaktiken (vgl. z.B. Kuntze \& Reiss 2006; Imgrund \& Radisch, 2014).

Neben einigen der historisch ausdifferenzierten theoretischen Grundlagen, die für die Umsetzung einer textbasierten Kasuistik galten und gelten (z.B. Soziokonstruktivismus, case-based reasoning, Anchored-Instruction Ansatz, Cognitive-Apprenticeship-Ansatz), können für die Arbeit mit Unterrichtsvideos noch das situierte Lernen bzw. die situierte Kognition ergänzend genannt werden (vgl. Greeno 1998). 


\section{Zweiter Ordnungsversuch: Ziele videobasierter Kasuistik - Was ist der Fall?}

Grundsätzlich lassen sich in der Lehrer*innenbildung und -fortbildung drei Ziele der videobasierten Kasuistik unterscheiden: Illustration, Kompetenzentwicklung und Rekonstruktion. Je nach Zielsetzung unterscheidet sich auch die Bestimmung des Falles (siehe unten). Klar im Mittelpunkt der in diesem Beitrag fokussierten videobasierten Kasuistik steht das zweite Ziel: die Kompetenzentwicklung. Mit Unterrichtsvideos soll die eigene professionelle Unterrichtswahrnehmung der Lehramtsstudierenden und Lehrkräfte geschult werden (vgl. Schneider u.a. 2016). Je nach theoretischer Anbindung wird diese Kompetenz zwar unterschiedlich benannt, im Kern geht es jedoch nahezu durchgängig um das Erkennen von relevanten Situationen im Video sowie die schrittweise Analyse dieser (vgl. z.B. Sherin 2007; Janík \& Seidel 2009; Sherin \& van Es 2009; Tripp \& Rich 2012; Seidel \& Stürmer 2014). Eng verknüpft mit diesem zweiten Ziel ist auch die Selbst- und Fremdevaluation, die davon ausgehen, dass in Unterrichtsvideos Aspekte eigenen und fremden Handelns beschrieben und beurteilt werden können. Die Bestimmung des Falles in der videobasierten Kasuistik gestaltet sich je nach Zielsetzung unterschiedlich. Grundsätzlich gilt jedoch für alle Ziele, dass im Video alles zum Fall gemacht werden kann, was bedeutet, dass in den authentischen Handlungssituationen sowohl Problemkonstellationen betont werden können (vgl. Kade 1990; Messmer 2001) oder aber genau das Alltägliche und Unspektakuläre in den Fokus gerückt werden kann. Für das Ziel der Illustration wird hierfür im Video ein Ausschnitt bestimmt, der exemplarisch für ein Phänomen steht. Exemplarisch verweist hierbei darauf, dass sich pädagogische Phänomene im Fall widerspiegeln, die stellvertretend für weitere Probleme und Herausforderungen des Unterrichtsalltags stehen (vgl. Goeze \& Hartz 2010). Für das Ziel der Kompetenzentwicklung muss bei der Fallbestimmung nach dem didaktischen Vorgehen beim Kompetenzerwerb unterschieden werden (siehe auch Kap. 4.2): Ausschnitte bzw. Sequenzen des Videos oder das ganze Unterrichtsvideo selbst können zum Fall durch den Dozierenden gemacht werden, an dem die Studierenden etwas wiedererkennen und analysieren sollen (deduktiv) oder die Studierenden sollen im Video selbst Aspekte erkennen/identifizieren und analysieren (induktiv). Im Falle des Ziels der Rekonstruktion, die jedoch mit Unterrichtsvideos bisher selten in der Lehrer*innenbildung und -fortbildung zum Einsatz kommt (siehe auch Kap. 6), ergibt sich der Fall erst durch die Arbeit mit dem Unterrichtsvideo. Einschränkend sei für alle drei Ziele erwähnt, dass im Video nur zum Fall gemacht werden kann, was auch aufgenommen wurde. Bereits bei der Videoaufzeichnung ergeben sich bspw. durch Kameraperspektive und Kamerafokus Einschränkungen, die dazu führen, dass nicht das ganze Unterrichtsgeschehen sichtbar und damit zum Fall machbar ist. 
Aus inhaltlicher Sicht fokussiert die videobasierte Kasuistik im kompetenztheoretischen Sinne in der Regel das Kerngeschäft des Lehrer*innenhandelns und macht dies zum Fall: das Unterrichten. Möglichkeiten bieten sich jedoch auch für die Thematisierung von Erziehungs- und Beurteilungshandeln. Seltener stehen andere Aspekte im Fokus der videobasierten Kasuistik, wie z.B. das Thema Elterngespräche (vgl. Gartmeier 2014). In dieser Auflistung verdeutlicht sich nochmals der zugrundeliegende kompetenztheoretische Zugang: Illustriert oder analysiert werden in der Regel Aspekte eines kompetenzorientierten Zugriffs auf den Lehrer*innenberuf.

\section{Dritter Ordnungsversuch: Wirkungen videobasierter Kasuistik in der Lehrer*innenbildung}

Folgt man dem zweitgenannten Ziel videobasierter Kasuistik in der Lehrer*innenbildung, so sollen bei Studierenden bzw. Lehrkräften Kompetenzen in der professionellen Wahrnehmung von Unterricht geschult werden. Empirische Ergebnisse hierzu (auch im Vergleich zur textbasierten Kasuistik) werden im Kapitel 3.1 vorgestellt. Da der Kompetenzerwerb eng mit weiteren kognitiven sowie motivational-affektiven Prozessen verknüpft ist, die sich auf den Lernerfolgt mit Unterrichtsvideos auswirken, werden empirische Studien hierzu im Kapitel 3.2 zusammenfassend dargestellt.

\subsection{Effekte auf die professionelle Wahrnehmung von Unterricht}

Auf den ersten Blick scheint der Einsatz von Unterrichtsvideos gegenüber Fällen in schriftlicher Form für die Lehrer*innenbildung viele Vorteile aufzuweisen (vgl. Krammer 2014). Die Komplexität unterrichtlichen Handelns lässt sich umfassender darstellen, da mehr Informationen als beim bloßen Erinnern oder schriftlichen Fixieren verfügbar sind. Damit kann eine Übervereinfachung durch Mehrdimensionalität und Multimodalität vermieden werden (vgl. Spiro u.a. 1988, 378). Dies kann jedoch auch zum Problem werden, wenn beispielsweise durch die hohe Informationsdichte wesentliche Details nicht erkannt werden (vgl. Dinkelaker \& Herrle 2009, 41ff.). Videobasierte Kasuistik ermöglicht eine handlungsdruckbefreite und damit auch systematische Arbeit am Fall (vgl. Reusser 2005, 10), bei der auch temporale und emotionale Aspekte besser als im Text dargestellt werden können (vgl. Moreno \& Valdez 2007, 194f.). Zudem wird eine nicht-lineare und auch wiederholte Betrachtung (vgl. Brouwer 2011, 2) ermöglicht. Videofälle versprechen eine hohe Authentizität (vgl. Hatch \& Grossmann 2009) und vermitteln dadurch Realitätsnähe (vgl. Spiro u.a. 2007, 95f.). 


\section{Marcus Syring}

In den letzten 15 Jahren gab es zahlreiche Forschungsbemühungen zur Frage der Effektivität videobasierter Kasuistik, vor allem mit Blick auf die Analyse von Unterricht (vgl. zusammenfassend auch die Überblicke bei Gaudin \& Chaliès 2015 sowie Steffensky \& Kleinknecht 2016). So zeigen die Ergebnisse von Interventionsstudien über die Teilnahme an video clubs von Sherin (2007) bzw. van Es und Sherin (2008), dass Lehrkräfte zunehmend thematisch fokussiertere Selektionen von relevanten Unterrichtssequenzen vornehmen. Dass nicht nur die Selektion, sondern auch die Qualität der Analyse bzw. Erörterung relevanter Situationen sich durch die videobasierte Kasuistik steigern lässt, zeigen die Ergebnisse der Pilotierungsstudie von Gold u.a. (2013): Zwar lassen sich keine Unterschiede zur textbasierten Gruppe bei ihnen finden, aber sowohl die video- als auch textbasierte Gruppe bewegten sich durch die Fallarbeit in Richtung eines Expert*innenratings der Fälle.

Oftmals werden video- und textbasierte Fallarbeit hinsichtlich ihrer Effektivität mit Blick auf die Steigerung der Kompetenz zur professionellen Unterrichtswahrnehmung verglichen. So untersuchten Moreno und Valdez (2007) die Wirksamkeit von Video- und Textfällen zur Exemplifizierung pädagogischer Prinzipien. Sie fanden einen Vorteil für Videofälle direkt nach dem Treatment, der Effekt verschwand aber bei erneuter, zeitverzögerter Testung (vgl. ebd., 198-200). Moreno und Ortegano-Layne konnten diese Ergebnisse später replizieren (2008, 457460). Sunder u.a. (2016) verglichen die professionelle Wahrnehmung mit Blick auf das Thema Lernunterstützung zwischen textbasierten Fällen und textbasierten Fällen, die durch Videos angereichert waren. Sie zeigen, dass die Förderung der themenspezifischen professionellen Wahrnehmung in der letzteren Gruppe erfolgreicher war. Zu einem etwas anderen Bild kamen Schneider u.a. (2016) in ihrer Pre-Post-Interventionsstudie, in der video- und textbasierte Lehr-Lernarrangements mit Lehramtsstudierenden zum Thema Classroom Management verglichen wurden. Hier ergab sich ein signifikanter Unterschied für die Qualität der Erörterungen zugunsten der Textfälle (vgl. ebd., 484-486).

Die uneindeutige Befundlage erscheint unbefriedigend. Hier setzt auch die Kritik von Baecher u.a. (2018) an: Die Lehr-Lernarrangements sind zwischen den Studien sehr unterschiedlich und in vielen Artikeln auch nur schwer nachvollziehbar. Damit erscheinen sie nicht replizierbar und somit bleibt die Evidenz auch nur schwer vergleichbar. Zudem zeigen Yadav u.a. (2011, 25-31) in ihrer Studie eine signifikante Interaktion zwischen dem gewählten Fallmedium und dem Inhalt. Video- oder Textfälle sind also nicht per se besser oder schlechter, sondern die jeweiligen Vor- und Nachteile ergeben sich erst in der Kombination mit einem bestimmten Inhalt. Es erscheint also bedeutend für den Lernerfolg, zunächst die thematische Passung zum Fallmedium zu klären. 


\subsection{Effekte auf kognitive und motivational-affektive Prozesse}

Im Bereich der videobasierten Kasuistik lassen sich einige Forschungsbefunde zur Motivation und den Emotionen beim Lernen finden, da diese wichtige personenbezogene Determinanten für erfolgreiches Lernen darstellen. So stellen Moreno und Ortegano-Layne fest: „affective factors mediate learning by increasing or decreasing cognitive engagement" $(2008,461)$. Die Ergebnisse vieler Studien zeigen, dass Videofälle - nicht nur, aber auch im Vergleich zu Textfällen - als motivierend erachtet werden (vgl. Roth 2007), eine hohe Akzeptanz genießen (vgl. Lefstein \& Snell 2011; Yadav u.a. 2011; Kleinknecht \& Schneider 2013) und als befriedigend empfunden werden (vgl. Krammer u.a. 2010). Dies führt dazu, dass Studienteilnehmer*innen ein höheres Engagement beim Lernen mit Videofällen berichten (vgl. Petermandl 1982; Yadav u.a. 2011). Keine motivationalen Unterschiede fanden Sunder u.a. (2016, 349-351) beim Vergleich von Textfällen und einer Kombination aus Text- und Videofällen. Gar gegenteilige Ergebnisse berichten Goldman (2007), der eine höhere Involviertheit der Studierenden bei Textfällen fand, und Koehler u.a. (2005), die ein höheres berichtetes Interesse bei Textfällen feststellen. Syring u.a. (2015a, 676f.) zeigen in ihrer Studie, dass videobasierte Fallarbeit bei Novizen im Vergleich zu Textfällen zu einer erhöhten kognitiven Belastung führt. Dies kann damit zusammenhängen, dass Textfälle eine höhere Komplexitätsreduktion aufweisen (vgl. Birdwhistell 1970) und sequentiell vorliegen. Gold u.a. (2016) kommen allerdings zum gegenteiligen Ergebnis. Weiterhin berichten Syring u.a. (2015, 677-679), ebenso wie Gold u.a. (2016), dass es in den Interventionen keinen motivationalen Unterschied zwischen video- und textbasierten Seminaren gab. Auch das Interesse an der Fallarbeit war in beiden Gruppen gleich. Lediglich bei der fallbasierten Hausaufgabe berichten Syring u.a. (2015a, 678) eine geringere Motivation bei der Arbeit mit Videos. Hinsichtlich der Emotionen zeigen sie ferner, dass bei der videobasierten Fallarbeit mehr Freude, bei der textbasierten hingegen mehr Ärger berichtet wird. Beide Valenzen können aber als lernförderlich angenommen werden. Unklar bleibt, ob sich die berichteten Emotionen auf die Arbeit mit den Fällen, das gewählte Fallmedium oder die in den Fällen verhandelten Themen respektive Inhalte beziehen. Die Studien von Koehler u.a. (2005) sowie von Moreno und Valdez (2007) bzw. Moreno und Ortegano-Layne (2008) weisen ebenfalls auf eine höhere emotionale Beteiligung der Studierenden bei der Arbeit mit Videofällen hin. 


\section{Vierter Ordnungsversuch: Merkmale von videobasierten Lernumgebungen}

Für den Lernerfolg bei der Arbeit mit Unterrichtsvideos spielen unterschiedliche Aspekte eine Rolle (vgl. Santagata u.a. 2007; Borko u.a. 2008; Krammer u.a. 2008; Hatch \& Grossman 2009). So lassen sich mit Blick auf die Gestaltung der Lernumgebung unterschiedliche didaktische Variationsmöglichkeiten nennen. Grundsätzlich lässt sich die videobasierte Fallarbeit nach den Videogeber*innen (eigene vs. fremde Videos) sowie nach dem Lehr-Lernmodell (instruktional vs. problemorientiert) unterscheiden. Empirische Ergebnisse hierzu werden in den Kapiteln 4.1 und 4.2 präsentiert. Weitere Möglichkeiten der Unterscheidung der Lernumgebungen betreffen die Art der Lernaufgaben (offen vs. strukturiert/ fokussiert) sowie die Arbeits- und Sozialform (individuell vs. kollaborativ) (vgl. z.B. Borko u.a. 2011; Blomberg u.a. 2013).

\subsection{Effekte unterschiedlicher Varianten von Videos}

Für den Einsatz eigener Videos in der Fallarbeit sprechen sich Sherin (2004) und Borko u.a. (2008) aus. Sherin berichtet in ihrem Beitrag, dass eigene Videos die gezielte Reflexion von Lehr- und Lernprozessen in der eigenen Klasse ermöglichen. Borko betont, dass sich die eigenen Videos besonders gut eignen, da man sich aufgrund des Wissens um den Kontext besser in die Situation eindenken könne. Hinzu kommt, dass eigene Videos gezielt zur eigenen professionellen Entwicklung herangezogen werden können. Dinge, die man im eigenen Unterricht nicht wahrnimmt (da man aufgrund von Handlungsdruck nicht reflektieren kann), können so aus der Distanz heraus gedeutet und nutzbar gemacht werden (vgl. Harlin 2014).

Seago (2004) vermutet hingegen, dass sich besonders fremde Videos dazu eignen, neue fachliche und (fach-)didaktische Inhalte zu erlernen, weswegen diese auch eher in Lehrerfortbildungen zum Einsatz kommen. Hier kann auch durch die emotionale Distanz (vgl. Seago 2004) Reflexion besser eingeübt und eine Diskussion über Handlungsalternativen angeregt werden (vgl. Borko u.a. 2011).

Unterschiedliche Studien vergleichen explizit den Einsatz eigener und fremder Unterrichtsvideos. Seidel u.a. (2011, 264f.) berichten eine positive Wirkung eigener Videos auf die Motivation und die kognitiven Prozesse bei der Fallarbeit. Ebenso fanden sie eine häufigere Aufmerksamkeit auf lernrelevante Merkmale bei den Schüler*innen bzw. Lehrkräften im Kontrast zu beiläufigen oder irrelevanten Aspekten im Video. Im Gegensatz dazu identifizieren sie bei fremden Videos eine intensivere Analyse v.a. problematischer Stellen (es wurden häufiger Handlungsalternativen benannt). Das Benennen von mehr Handlungsalternativen bei fremden Videos fanden auch Kleinknecht und Schneider $(2013,18-20)$ in ihrer Studie. Zudem berichten sie deutlich mehr negative und etwas mehr positive Emotionen 
bei fremden Videos. Vertiefende Analysen zeigen hier, dass die Emotion Enttäuschung bei fremden Videos mit einer vertieften Analyse der Handlung einhergeht und negative Emotionen bei eigenen Videos mit einer Abwehr- und Rechtfertigungshaltung (vgl. Kleinknecht \& Poschinski 2014, 480-484). Zhang u.a. (2011) zeigen, dass Lehrkräfte ihre eigenen Videos als am besten geeignet zum Lernen einschätzen. Krammer u.a. (2016) berichten in ihrer Studie, dass gegenüber einer Kontrollgruppe sowohl bei eigenen als auch fremden Videos ein Anstieg der professionellen Wahrnehmung zu verzeichnen ist, aber kein Unterschied zwischen den Gruppen zu finden ist.

\subsection{Effekte unterschiedlicher Lehr-Lernmodelle mit Videos}

Mayer u.a. $(2009,53)$ stellten in einem Aufsatz fest: „media do not cause learning, methods cause learning “. Damit stellt sich die Frage nach den Effekten der methodischen Einbettung videobasierter Fallarbeit. Hierzu findet sich eine breite Variation an Einsatzmöglichkeiten (vgl. Orland-Barak \& Maskit 2017), was auch den Vergleich unterschiedlicher Interventions- und Evaluationsstudien schwierig macht (vgl. Baecher u.a. 2018). Im Folgenden soll daher exemplarisch nur auf die häufige Unterscheidung eher instruktionaler (starke Steuerung durch einen Dozierenden) versus eher problembasierter Lehr-Lernarrangements (starke Selbstbestimmung durch Beobachter*in) eingegangen werden (vgl. Kleinknecht u.a. 2014). Damit werden in der videobasierten Kasuistik zwei zentrale Konzepte der didaktisch-methodischen Planung und Durchführung von Lehrveranstaltungen aufgegriffen (vgl. Oser \& Baeriswyl 2001). Das problembasierte Vorgehen wird häufig als diskursiver und motivierender charakterisiert, kann aber auch überfordern bzw. zu hohen kognitiven Belastungen führen (vgl. Kirschner u.a. 2006). Dem instruktionalen Vorgehen wird zugeschrieben, die fokussierte Einübung eines Analyseprozesses besser zu unterstützen, auch aufgrund einer kognitiven Entlastung durch den Dozierenden (vgl. Santagata \& Guarino 2011).

Seidel u.a. (2013) verglichen in einer Interventionsstudie bei Lehramtsstudierenden zwei Gruppen: Eine Gruppe, die videofallbasiert eher deduktiv-angeleitet (Rule-Example) arbeitete und eine Gruppe, die eher induktiv-problembasiert (Example-Rule) arbeitete. Sowohl beim deklarativen Wissen als auch bei der Analysekompetenz (hier als professional vision bezeichnet) schnitt die deduktive Gruppe besser ab. Beim Test des Transfers des Wissens jedoch, welcher über die Planung einer Unterrichtsstunde erfasst wurde, schnitt die induktive Gruppe besser $a b$ (vgl. ebd., 61f.). Syring u.a. (2015b) sowie Schneider (2016) verglichen zwei videobasierte Seminartypen miteinander: ein instruktionales Setting und ein problembasiertes. Die Ergebnisse dieser Studien zeigen, dass die kognitive Belastung in beiden Gruppen über die Zeit hinweg abnahm und die Motivation anhaltend hoch blieb. Die Involviertheit und das Engagement schätzten die Lehramtsstudierenden in der problemorientierten Gruppe höher ein. Ebenso empfanden sie 
mehr Freude bei der problemorientierten Vorgehensweise (vgl. Syring u.a. 2015b, 122-124). Mit Blick auf die Analysekompetenz verbesserte sich die theoretische Fundiertheit der Analysen in beiden Seminartypen (vgl. Schneider 2016, 67). Für ein eher instruktionales Vorgehen sprachen sich auch Studierende in einer Interviewstudie von Brouwer und Robijens (2013) aus: Sie berichten eine höhere Zufriedenheit und einen höheren Lernerfolg bei einer stärkeren Prozessbegleitung.

\section{Didaktische Empfehlungen}

Fasst man die empirischen Ergebnisse zusammen, so ergibt sich kein einheitliches Bild (vgl. Steffensky \& Kleinknecht 2016). Zwar werden viele Vorteile einer videobasierten Kasuistik in Studien belegt, jedoch ergibt sich nicht unbedingt ein Vorteil gegenüber einer textbasierten Fallarbeit. Bei der Planung eines fallbasierten Seminars bedarf es in jedem Einzelfall einer genauen didaktischen wie methodischen Überprüfung. Kleinknecht u.a. (2014) empfehlen daher für die Planung videobasierter kasuistischer Seminare folgendes Vorgehen (vgl. auch Syring 2016):

- Lehr-Lernarrangement auswählen und planen: Je nach Lernziel sollte ein passendes Lehr-Lernarrangement gewählt werden. Geht es vorrangig um die Schulung diskursiv-argumentativer Fähigkeiten sowie um die Planung und Reflexion von Unterricht, so empfiehlt sich ein problembasiertes Vorgehen. Soll stärker ein analytisches Vorgehen eingeübt oder pädagogisches und (fach-)didaktisches Wissen vermittelt werden, empfiehlt sich ein instruktionaleres Vorgehen.

- Videotyp auswählen und kategorisieren: Bietet sich die Arbeit mit eigenen Videos an, um auch aufgrund der Kontextinformationen näher am realen Geschehen zu sein oder lohnen sich eher fremde Videos, die häufiger aufgrund der Distanz gehaltvoller analysiert werden? Ebenfalls sollte geprüft werden, inwiefern der zu transportierende Gegenstand/ Inhalt zum Videoformat passt.

- Fremde Videos auswählen und kontextualisieren: Mittlerweile finden sich viele Onlineplattformen, auf denen man Zugriff auf fremde Unterrichtsvideos erhält (eine Übersicht findet sich bei Petko u.a. 2014). Wichtig ist dabei - aus der Perspektive eines kompetenzorientierten Ansatzes - die kontextuelle Einbettung der Videos, da die Analysen sonst schnell im Ungefähren verlaufen.

- Für eigene Videos Lernumgebung sorgfältig planen: Eigene Videos bergen die Gefahr, dass die Fallgeber*in schnell in einen Rechtfertigungsmodus verfällt und die Kolleg*innen bzw. Kommiliton*innen sich ggf. nicht trauen, kritische Aspekte im Fall zu benennen, um die Beziehungsebene nicht zu gefährden. Daher sollte die Lernumgebung hier sorgfältig geplant (z.B. Schaffen von Vertrauen in der Gruppe, Einführung von Feedback-Regeln etc.) und die Fallarbeit gut begleitet bzw. moderiert (z.B. Achten auf Wertfreiheit in der Analyse, Bestärkung der Fallgeber*in etc.) werden. 
Neben den genannten Aspekten sind weitere zu berücksichtigen: Bei der genauen Planung von videobasierten Fallseminaren sind auch die Einstellungen der Dozierenden zur Fallarbeit selbst ein entscheidender Faktor. Schneider u.a. (2016) konnten einen starken Einfluss der Einstellung der Dozierenden gegenüber dem verwendeten Fallmedium auf die Verwendung theoretischer Prinzipien in der Fallarbeit nachweisen. Ein weiterer Punkt betrifft die Dauer von videobasierten Fallseminaren. Hier zeigten Studien, dass der Effekt des Videoeinsatzes in der Fallarbeit auf die Analysekompetenz schon in relativ wenigen Sitzungen sichtbar wird (vgl. Kleinknecht u.a. 2014).

\section{Zusammenfassung und Ausblick}

In den hier erarbeiteten Ordnungsversuchen ist eine enge Verknüpfung der videobasierten Kasuistik mit dem kompetenzorientierten Professionsansatz herausgearbeitet geworden (vgl. Tab. 1). Dies mag auch damit zusammenhängen, dass in der Unterrichts- und Schulforschung mit Videos das quantitative Paradigma überwiegt (vgl. z.B. Seidel \& Thiel 2017), welches eine hohe Affinität zum kompetenzorientierten Ansatz aufweist. Jedoch gibt es zunehmend auch qualitative und rekonstruktive Arbeiten, die sich Unterrichtsvideos bedienen (z.B. die dokumentarische Methode; siehe für die Erziehungswissenschaft: Asbrand \& Martens 2018 bzw. für die fachdidaktische Forschung: Tesch 2019). Hier gilt es noch Möglichkeiten und didaktische Varianten zu entwickeln und ggf. empirisch zu prüfen, wie auch diese in der Lehrer*innenbildung fruchtbar gemacht werden können.

Tab. 1: Systematisierung der vier Ordnungsversuche

\begin{tabular}{ll}
\hline \multicolumn{1}{c}{ Ordnungsversuch } & \multicolumn{1}{c}{ Kategorien } \\
\hline Historische Entwicklung videobasierter & - Microteaching / Interaction Analysis \\
Kasuistik & - Modelling Expert Teaching \\
& - video-based cases \\
& - Unterrichtsmitschau \\
\hline Ziele videobasierter Kasuistik - Was ist der & - Illustration \\
Fall? & - Kompetenzerwerb \\
& - Reflexion \\
\hline Wirkungen videobasierter Kasuistik in der & - Kompetenzerwerb: (Professionelle Wahr- \\
Lehrer*innenbildung & nehmung von Unterricht) \\
& - Kognitive und motivational-affektive \\
& Prozesse beim Lernen \\
\hline
\end{tabular}




\begin{tabular}{lc}
\hline \multicolumn{1}{c}{ Ordnungsversuch } & Kategorien \\
\hline Merkmale von videobasierten & - Videogeber*in (eigene vs. fremde Videos) \\
Lernumgebungen & - Lehr-Lernmodell (instruktional vs. pro- \\
& blemorientiert) \\
& - Lernaufgaben (offen vs. strukturiert) \\
& - Arbeits- und Sozialform (eigenständig vs. \\
& Gruppenarbeit) \\
\hline
\end{tabular}

Künftige Forschung sollte sich weiter mit der Frage beschäftigen, wie unterschiedliche Videoformate in der Lehrer*innenbildung eingesetzt werden können und welche Effekte diese zeigen. So ist noch nicht geklärt, ob beispielsweise eher Lehrkraft- oder Klassenkameras sinnvoll sind oder gar multiple Aufnahmen im Klassenzimmer, z.B. über kleine Kameras an jedem Tisch. Ebenso muss sich weitere Forschung darum bemühen, stärker darauf zu schauen, ob und wie sich die tatsächliche Praxis von Lehrkräften durch die Nutzung videobasierter Kasuistik verändert. Erste Ergebnisse zeigen bereits, dass sich diese Veränderung bis in Klassenzimmer hinein auswirken kann (vgl. z.B. Santagata \& Yeh 2014; Sun \& van Es 2015).

\section{Literaturverzeichnis}

Asbrand, B. \& Martens, M. (2018): Dokumentarische Unterrichtsforschung. Wiesbaden: Springer VS.

Baecher, L., Kung, S.-C., Ward, S.L. \& Kern, K. (2018): Facilitating Video Analysis for Teacher Development: A Systematic Review of the Research. In: Journal of Technology and Teacher Education 26 (2), 185-216.

Baumert, J. \& Kunter, M. (2006): Stichwort professionelle Kompetenz von Lehrkräften. In: Zeitschrift für Erziehungswissenschaft 9 (4), 469-520.

Birdwhistell, R.L. (1970): Kinesics and context: Essays on body motion communication. Philadelphia: University of Pennsylvania Press.

Blomberg, G., Renkl, A., Sherin, M.G., Borko, H. \& Seidel, T. (2013): Five research-based heuristics for using video in pre-service teacher education. In: Journal of Educational Research Online, 5 (1), 90-114.

Borko, H., Jacobs, J.K., Eiteljorg, E. \& Pittman, M.E. (2008): Video as a tool for fostering productive discussions in mathematics professional development. In: Teaching and Teacher Education 24 (2), 417-436.

Borko, H., Koellner, K., Jacobs, J. \& Seago, N. (2011): Using video representations of teaching in practice-based professional development programs. In: ZDM 43 (1), 175-187.

Brouwer, N. \& Robijens, F. (2013): Fokussierte Auswertung von Videoaufzeichnungen als Methode in der Lehrerausbildung. In: U. Riegel \& K. Macha (Hrsg.): Videobasierte Kompetenzforschung in den Fachdidaktiken. Münster u.a.: Waxmann, 302-318.

Brouwer, N. (2011): Imaging teacher learning, Paper presented at the AERA Annual Meeting in New Orleans.

Dinkelaker, J. \& Herrle, M. (2009): Erziehungswissenschaftliche Videographie. Eine Einführung. Wiesbaden: Springer VS. 
Dorlöchter, H., Krüger, U. \& Wiebusch, D. (2013): Videografie in der Lehrer(aus)bildung. Ein orientierender Überblick. In: Seminar 19 (2), 6-8.

Gartmeier, M. (2014): Fiktionale Videofälle in der Lehrerinnen- und Lehrerbildung. In: Beiträge zur Lehrerinnen- und Lehrerbildung, 32. Jg., 265-246.

Gaudin, C. \& Chaliès, S. (2015): Video viewing in teacher education and professional development: A literature review. In: Educational Research Review, 16. Jg., 41-67.

Goeze, A. \& Hartz, S. (2010): Lehrende lernen am Fall. Konzepte fallbasierten Lernens von der Weiterbildung bis zur Frühpädagogik. In: J. Schrader, R. Hohmann \& S. Hartz (Hrsg.): Mediengestützte Fallarbeit. Konzepte, Erfahrungen und Befunde zur Kompetenzentwicklung von Erwachsenenbildnern. Bielefeld: W. Bertelsmann, 101-124.

Gold, B., Förster, S. \& Holodynski, M. (2013): Evaluation eines videobasierten Trainingsseminars zur Förderung der professionellen Wahrnehmung von Klassenführung im Grundschulunterricht. In: Zeitschrift für Pädagogische Psychologie 27 (3), 141-155.

Gold, B., Hellermann, C., Burgula, K. \& Holodynski, M. (2016): Fallbasierte Unterrichtsanalyse. Effekte von video- und textbasierter Fallanalyse auf kognitive Belastung, aufgabenspezifisches Interesse und die professionelle Unterrichtswahrnehmung von Grundschullehramtsstudierenden. In: Unterrichtswissenschaft 44 (4), 322-338.

Goldman, R. (2007): Video representations and the perceptivity framework: epistemology, ethnography, evaluation, and ethics, In: R. Goldman, R. Pea, B. Barron \& S.J. Derry (Hrsg.): Video research in the learning sciences. Mahwah: Lawrence Erlbaum, 383-395.

Greeno, J.G. (1998): The situativity of knowing, learning, and research. In: American Psychologist, 53. Jg., 5-26.

Harlin, E.-M. (2014): Watching oneself teacher long-term effects of teachers' reflections on their video-recorded teaching. In: Technology, Pedagogy and Education 23 (4), 507-521.

Hatch, T. \& Grossman, P. (2009): Learning to Look beyond the Boundaries of Representation: Using Technology to Examine Teaching. In: Journal of Teacher Education 60 (1), 70-85.

Imgrund, B. \& Radisch, F. (2014): Praxisorientierte Unterrichtsforschung und Unterrichtsentwicklung in der Fachdidaktik: Videobasierte Fallstudien und Fallsammlungen - ein Beispiel aus der Fremdsprachendidaktik mit Checkliste für fachdidaktische Projekte. In: Beiträge zur Lehrerinnenund Lehrerbildung 32 (2), 262-274.

Janík, T. \& Seidel, T. (2009): The power of video studies in investigating teaching and learning in the classroom. Münster u.a.: Waxmann.

Kade, S. (1990): Handlungshermeneutik. Qualifizierung durch Fallarbeit. Bad Heilbrunn: Klinkhardt.

Kirschner, P.A., Sweller, J. \& Clark, R.E. (2006): Why Minimal Guidance During Instruction Does Not Work: An Analysis of the Failure of Constructivist, Discovery, Problem-Based, Experiential, and Inquiry-Based Teaching. In: Educational Psychologist 41 (2), 75-86.

Kleinknecht, M. \& Poschinski, N. (2014): Eigene und fremde Videos in der Lehrerfortbildung. Eine Fallanalyse zu kognitiven und emotionalen Prozessen beim Beobachten zweier unterschiedlicher Videotypen. In: Zeitschrift für Pädagogik 60 (3), 471-490.

Kleinknecht, M. \& Schneider, J. (2013): What do teachers think and how do they feel when they analyze videos of themselves teaching and of other teachers teaching? In: Teaching and Teacher Education, 33. Jg., 13-23.

Kleinknecht, M., Schneider, J. \& Syring, M. (2014): Varianten videobasierten Lehrens und Lernens in der Lehrpersonenaus- und -fortbildung - Empirische Befunde und didaktische Empfehlungen zum Einsatz unterschiedlicher Lehr-Lern-Konzepte und Videotypen. In: Beiträge zur Lehrerinnenund Lehrerbildung 32 (2), 210-220. 
Koehler, M.J., Yadav, A., Phillips, M.M. \& Cavazos-Kottke, S.C. (2005): What is video good for? Examining how media and story genre interact. In: Journal of Educational Multimedia and Hypermedia 14 (3), 249-272.

Krammer, K. (2014): Fallbasiertes Lernen mit Unterrichtsvideos in der Lehrerinnen- und Lehrerbildung. In: Beiträge zur Lehrerinnen- und Lehrerbildung 32 (2), 164-175.

Krammer, K., Hugener, I., Biaggi, S., Frommelt, M., Fürrer Auf der Maur, G. \& Stürmer, K. (2016): Videos in der Ausbildung von Lehrkräften: Förderung der professionellen Unterrichtswahrnehmung durch die Analyse von eigenen bzw. fremden Unterrichtsvideos. In: Unterrichtswissenschaft 44 (4), 257-372.

Krammer, K., Schnetzler, C., Pauli, C., Reusser, K., Lipowsky, F. \& Klieme, E. (2010): Unterrichtsvideos in der Lehrerfortbildung. Überblick über Konzeption und Ergebnisse einer einjährigen netzgestützten Fortbildungsveranstaltung. In: F.H. Müller, A. Eichenberger, M. Lüders \& J. Mayr (Hrsg.): Lehrerinnen und Lehrer lernen. Konzepte und Befunde zur Lehrerfortbildung. Münster u.a.: Waxmann, 227-243.

Krammer, K., Schnetzler, C.L., Ratzka, N., Reusser, K., Pauli, C., Lipowsky, F. \& Klieme, E. (2008): Lernen mit Unterrichtsvideos: Konzeption und Ergebnisse eines netzgestützten Weiterbildungsprojekts mit Mathematiklehrpersonen aus Deutschland und der Schweiz. In: Beiträge zur Lehrerbildung 26 (2), 178-197.

Kuntze, S. \& Reiss, K. (2006): Evaluational Research on a Video-Based In-Service Mathematics Teacher Training Project - Reported Instructional Practice and Judgements on Instructional Quality. In: J. Novotná, H. Moraová, M. Krátká \& N. Stehlíková (Hrsg.): Proceedings of the 30th Conference of the International Group for the Psychology of Mathematics Education (PME). Prag: Karls-Universität, 1-8.

Lefstein, A. \& Snell, J. (2011): Professional vision and the politics of teacher learning. In: Teaching and Teacher Education 27 (3), 505-514.

Mayer, R.E., Stull, A., DeLeeuw, K., Almeroth, K., Bimber, B., Chun, D., Bulger, M., Campbell, J., Knight, A. \& Zhang, H. (2009): Clickers in college classrooms: Fostering learning with questioning methods in large lecture classes. In: Contemporary Educational Psychology 34 (1), 51-57.

Messmer, R. (2001): Lernen aus Fallgeschichten in der Lehrerinnen- und Lehrerbildung. In: Beiträge zur Lehrerbildung 19 (1), 82-92.

Moreno, R. \& Ortegano-Layne, L. (2008): Do classroom exemplars promote the application of principles in teacher education? A comparison of videos, animations, and narratives. In: Educational Technology Research and Development 56 (4), 49-465.

Moreno, R. \& Valdez, A. (2007): Immediate and delayed effects of using a classroom case exemplar in teacher education: The role of presentation format. In: Journal of Educational Psychology 99 (1), 194-206.

Orland-Barak, L. \& Maskit, D. (2017): Methodologies of Mediation in Professional Learning. Springer International Publishing.

Oser, F. \& Baeriswyl, F. (2001): Choreographies of Teaching: Bridging Instruction to Learning. In: V. Richardson (Hrsg.): Handbook of Research on Teaching. Washington, D.C.: American Educational Research Association, 1031-1065.

Petermandl, M. (1982): Videoeinsatz in der beruflichen Aus- und Fortbildung. Berlin: Erich Schmidt Verlag.

Petko, D. \& Reusser, K. (2004): Praxisorientiertes E-Learning mit Video. In: A. Hohenstein \& K. Wilbers (Hrsg.): Handbuch E-Learning. Expertenwissen aus Wissenschaft und Praxis. Köln: Deutscher Wirtschaftsdienst, 1-22.

Petko, D., Prasse, D. \& Reusser, K. (2014): Online-Plattformen für die Arbeit mit Unterrichtsvideos: Eine Übersicht. In: Beiträge zur Lehrerinnen- und Lehrerbildung 32 (2), 247-261. 
Ramboll Management Consulting GmbH (2018): Qualitätsoffensive Lehrerbildung. Zwischenbericht der Evaluation. Online unter: https://de.ramboll.com/-/media/files/rde/management-consulting/ studien_handreichungen/qualitaetsoffensive_lehrerbildung_zwischenbericht_der_evaluation.pdf ?la $=$ de. (Abrufdatum: 21.01.2020).

Reusser, K. (2005): Situiertes Lernen mit Unterrichtsvideos. In: Journal für LehrerInnenbildung 5 (2), 8-18.

Roth, W.-M. (2007): Epistemic mediation: Video data as filters for the objectification of teaching by teachers. In: R. Goldman, R. Pea, B. Barron \& S.J. Derry (Hrsg.): Video research in the learning sciences. Mahwah: Lawrence Erlbaum, 367-382.

Santagata, R. \& Guarino, J. (2011): Using video to teach future teachers to learn from teaching. In: ZDM 43 (1), 133-145.

Santagata, R. \& Yeh, C. (2014): Learning to teach mathematics and to analyze teaching effectiveness: Evidence from a video- and practice-based approach. In: Journal of Mathematics Teacher Education, 17. Jg., 491-514.

Santagata, R., Zannoni, C. \& Stigler, J.W. (2007): The role of lesson analysis in pre-service teacher education: An empirical investigation of teacher learning from a virtual video-based field experience. In: Journal of Mathematics Teacher Education 10 (2), 123-140.

Schneider, J. (2016): Lehramtsstudierende analysieren Praxis. Ein Vergleich der Effekte unterschiedlicher fallbasierter Lehr-Lern-Arrangements. Online unter: doi.org/10.15496/publikation-13255. (Abrufdatum: 23.07.2020).

Schneider, J., Bohl, T., Kleinknecht, M., Rehm, M., Kuntze, S. \& Syring, M. (2016): Unterricht analysieren und reflektieren mit unterschiedlichen Fallmedien: Eine Untersuchung zur vermeintlichen Überlegenheit von Video gegenüber Text. In: Unterrichtswissenschaft 44 (4), 474-491.

Seago, N. (2004): Using videos as an object of inquiry for mathematics teaching and learning. In: J. Brophy (Hrsg.): Using video in teacher education. Amsterdam: Elsevier, 259-286.

Seidel, T. \& Stürmer, K. (2014): Modeling and measuring the structure of professional vision in pre-service teachers. In: American Educational Research Journal 51 (4), 739-771.

Seidel, T. \& Thiel, F. (2017): Standards und Trends der videobasierten Lehr-Lernforschung. In: Zeitschrift für Erziehungswissenschaft 20, 1-21.

Seidel, T., Blomberg, G. \& Renkl, A. (2013): Instructional strategies for using video in teacher education. In: Teaching and Teacher Education, 34. Jg., 56-65.

Seidel, T., Stürmer, K., Blomberg, G., Kobarg, M. \& Schwindt, K. (2011): Teacher learning from analysis of videotaped classroom situations. Does it make a difference whether teachers observe their own teaching or that of others? In: Teaching and Teacher Education 27 (2), 259-267.

Sherin, M.G. (2007): The development of teachers' professional vision in video clubs. In: R. Goldman (Hrsg.): Video research in the learning sciences. Mahwah: Lawrence Erlbaum, 383-395.

Sherin, M.G. \& van Es, E.A. (2009): Effects of Video Club Participation on Teacher's Professional Vision. In: Journal of Teacher Education 60 (1), 20-37.

Sherin, M.G. (2004): New perspectives on the role of video in teacher education. In: J. Brophy (Hrsg.): Using video in teacher education. Amsterdam: Elsevier, 1-27.

Shulman, J.H. (1992): Case methods in teacher education. New York, London: Teachers College Press.

Shulman, L.S. (1986): Those who understand: Knowledge growth in teaching. In: Educational Researcher 15 (1), 4-14.

Spiro, R.J., Collins, B.P. \& Ramchandran, A. (2007): Reflections on a post-Gutenberg epistemology of video use in ill-structured domains: Fostering complex learning and cognitive flexibility. In: R. Goldman, R. Pea, B. Barron \& S.J. Derry (Hrsg.): Video research in the learning sciences. Mahwah: Lawrence Erlbaum, 93-100. 
Spiro, R.J., Coulson, R.L., Feltovich, P.J. \& Anderson, D.K. (1988): Cognitive Flexibility Theory: Advanced Knowledge Acquisition in Ill-Structured Domains. In: Cognitive Science Society (Hrsg.): Tenth Annual Conference of the Cognitive Science Society. Hillsdale, N.J.: Lawrence Earlbaum, 375-383.

Steffensky, M. \& Kleinknecht, M. (2016): Wirkungen videobasierter Lernumgebungen auf die professionelle Kompetenz und das Handeln (angehender) Lehrpersonen. In: Unterrichtswissenschaft 44 (4), 305-321.

Sun, J. \& van Es, E.A. (2015): An exploratory study of the influence that analyzing teaching has on preservice teachers' classroom practice. In: Journal of Teacher Education, 66. Jg., 201-214.

Sunder, C., Todorova, M. \& Möller, K. (2016): Förderung der professionellen Wahrnehmung bei Bachelorstudierenden durch Fallanalysen. Lohnt sich der Einsatz von Videos bei der Repräsentation der Fälle? In: Unterrichtswissenschaft 44 (4), 339-356.

Syring, M. (2015): Unterrichtsvideos als Allheilmittel in der Lehrerbildung? Kognitive Belastung, Motivation und Emotionen bei der Arbeit mit video- und textbasierten Unterrichtsfällen. In: M. Schiefner-Rohs, C. Gomez Tutor \& C. Menzer (Hrsg.): Lehrer. Bildung. Medien. Herausforderung für die Entwicklung und Gestaltung von Schule. Hohengehren: Schneider Verlag, 57-69.

Syring, M., Bohl, T., Kleinknecht, M., Kuntze, S., Rehm, M. \& Schneider, J. (2016): Fallarbeit als Angebot - fallbasiertes Lernen als Nutzung. Empirische Ergebnisse zur kognitiven Belastung, Motivation und Emotionen bei der Arbeit mit Unterrichtsfällen. In: Zeitschrift für Pädagogik 62 (1), 86-108.

Syring, M., Bohl, T., Kleinknecht, M., Kuntze, S., Rehm, M. \& Schneider, J. (2015a): Videos oder Texte in der Lehrerbildung? Effekte unterschiedlicher Medien auf die kognitive Belastung und die motivational-emotionalen Prozesse beim Lernen mit Fällen. In: Zeitschrift für Erziehungswissenschaft 18 (4), 667-685.

Syring, M., Kleinknecht, M., Bohl, T., Kuntze, S., Rehm, M. \& Schneider, J. (2015b): How problem-based or direct instructional case-based learning environments influence pre-service teachers' cognitive load, motivation and emotions: A quasi-experimental intervention study in teacher education. In: Journal of Education and Human Development 4 (4), 115-129.

Tesch, B. (2019): Sinnkonstruktion im Fremdsprachenunterricht. Einführung in die rekonstruktive Fremdsprachenforschung mit der dokumentarischen Methode. Berlin: Peter Lang.

Tripp, T. \& Rich, P. (2012): Using video to analyze one's own teaching. In: British Journal of Educational Technology 43 (4), 678-704.

van Es, E.A. \& Sherin, M.G. (2008): Mathematics teachers' "learning to notice" in the context of a video club. In: Teaching and Teacher Education 24 (2), 244-276.

Yadav, A., Phillips, M.M., Lundeberg, M.A., Koehler, M.J., Hilden, K. \& Dirkin, K.H. (2011): If a picture is worth a thousand words is video worth a million? Differences in affective and cognitive processing of video and text cases. In: Journal of Computing in Higher Education 23 (1), 15-37.

Zhang, M., Lundeberg, M.A., Koehler, M.J. \& Eberhardt, J. (2011): Understanding affordances and challenges of three types of video for teacher professional development. In: Teaching and Teacher Education 27 (2), 454-262. 